\title{
Aberrant Right Hepatic Artery with a Prepancreatic Course Visualized Prior to Pancreaticoduodenectomy
}

\author{
A. Venara • O. Pittet • T. L. Lu • N. Demartines • \\ N. Halkic
}

Received: 31 October 2012 / Accepted: 10 December 2012 /Published online: 4 January 2013

(C) 2012 The Society for Surgery of the Alimentary Tract

\begin{abstract}
Liver vascularization is known to present with several different variations. Generally, a normal vascular anatomy is reported in up to $50-80 \%$ of cases. For this reason, a precise preoperative mapping of the hepatic vascularization prior to pancreatic surgery is essential to avoid injuries and subsequent complications. We report here a case of a young patient scheduled for Whipple procedure, who presented an arterial pattern type Michels IV, variation reported in 0.6 to $3 \%$ in the literature. Another interesting particularity of this case was the fact that the right hepatic artery had a prepancreatic course. We think that every surgeon performing hepatopancreatic surgery should have heard of this special and rare situation.
\end{abstract}

Keywords Whipple procedure $\cdot$ Anatomy $\cdot$ Right hepatic artery $\cdot$ Liver vascularization

\section{Case Report}

A 37-year-old man with a past medical history of chronic pancreatitis with multiple episodes of acute recurrent pancreatitis was found to have a lesion of the ampulla of Vater. An endoscopic resection failed because the lesion was too large. Considering the size of the lesion, the presence of low-grade dysplastic areas and, most importantly, a long-term high risk of recurrence in a young patient, it was decided to treat the condition by Whipple procedure.

A. Venara $\cdot$ O. Pittet $\cdot$ N. Demartines $\cdot$ N. Halkic Department of Visceral Surgery, Centre Hospitalier Universitaire Vaudois, 46 rue du Bugnon, 1011 Lausanne, Switzerland

A. Venara $(\bowtie)$

Department of Visceral Surgery, Centre Hospitalier Universitaire, 4 rue Larrey, 49933 Angers, France

e-mail: auvenara@yahoo.fr

T. L. Lu

Department of Radiology, Centre Hospitalier Universitaire, 46 rue du Bugnon, 1011 Lausanne, Switzerland
The preoperative assessment included an angio-CT scan to define the anatomic relationships around the lesion. The CT scan identified an aberrant right hepatic artery (aRHA) arising from the superior mesenteric artery and following a prepancreatic route (Fig. 1a, b). Associated with this variant, an aberrant left hepatic artery arose directly from the left gastric artery (Fig. 1a).

During surgery, the hepatic pedicle was dissected, the pars flaccida of the omentum open, and a common hepatic artery was found, which was surprisingly not visible on the high-quality CT scan. This artery bifurcated near the hepatic pedicle into two arteries of larger caliber (one was in fact the aRHA) (Fig. 2a). Without the support of the preoperative imaging, this artery would have been misinterpreted as a large gastroduodenal artery in its normal anatomic position. By clamping the inferior part of the aRHA temporary, a reduced but still preserved blood flow was measured and therefore the artery was preserved. The intervention was continued normally by dissection of the retroportal plane, which was challenging due to the past episodes of pancreatitis. The aRHA was dissected along its whole course and, subsequently, the pancreatic head was liberated and the pancreas transected at the level of the isthmus while sliding the pancreatic parenchyma to the right of the artery. This maneuver freed the posterior part 
Fig. 1 CT scan reconstruction of celiac trunk and superior mesenteric artery - three quarters view (a) and front view (b) CT: Coeliac Trunk, ALHA: Aberrant left hepatic artery, ARHA : Aberrant right hepatic artery, LGA: Left gastric artery, SMA: Superior mesenteric artery
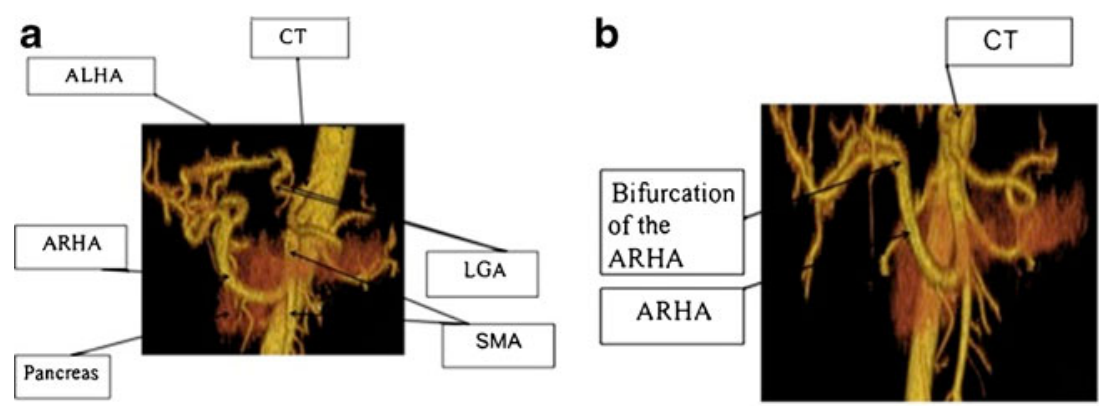

of the pancreas reducing the traction on the artery to a minimum and thus reducing the risks of vascular injury (Fig. 2b).

Finally, the pancreaticojejunostomy, the choledochojejunostomy, and the gastrojejunostomy could be performed without problems. The postoperative recovery was uneventful.

\section{Discussion}

Haller initially described anomalies of the arterial hepatic supply in $1756 .{ }^{1}$ Michels in $1966^{2}$ and then Hiatt in $1994^{3}$ proposed a classification of the various anatomic variants. So-called normal vascular configuration is observed in 50$80 \%$ of cases. ${ }^{2,4}$

In the present case, presenting with an aRHA arising from the superior mesenteric artery and an aberrant left hepatic artery originating from the left gastric artery, the anatomic variant corresponds to a type IV in both Michels and Hiatt classification ${ }^{2,3}$ (Fig. 2b). Additionally, a smallcaliber common hepatic artery was found arising from the celiac trunk and continued with the aRHA. If an aRHA from the superior mesenteric artery is the commonest variation (11 to $21 \%),{ }^{2-5}$ its association with an aberrant left hepatic artery is very rare $(0.6 \text { to } 3 \%)^{2,3}$ and has not been described up to now.

The key point of this case is the high risk of misinterpretation during surgery, which was minimized due to careful preoperative imaging showing the aberrant RHA. This could have been misinterpreted as a large-caliber gastroduodenal artery. Preoperative radiologic examination allows identifying the precise hepatic vascularization for better intraoperative management. ${ }^{6}$ Of note, angiography has been replaced by angio-MRI or angio-CT to assess vascularization. ${ }^{7}$ In particular, angio-CT is accurate
Fig. 2 Operating view of the Whipple procedure showing the prepancreatic course of the aberrant right hepatic artery and the aberrant left hepatic artery (a) and operating view after pancreatic resection (b). $L G A$ left gastric artery, a LHA aberrant left hepatic artery, $P$ pancreas, a RHA aberrant right hepatic artery, $V P$ portal vein, $V C$ vena cava, SMA superior mesenteric artery
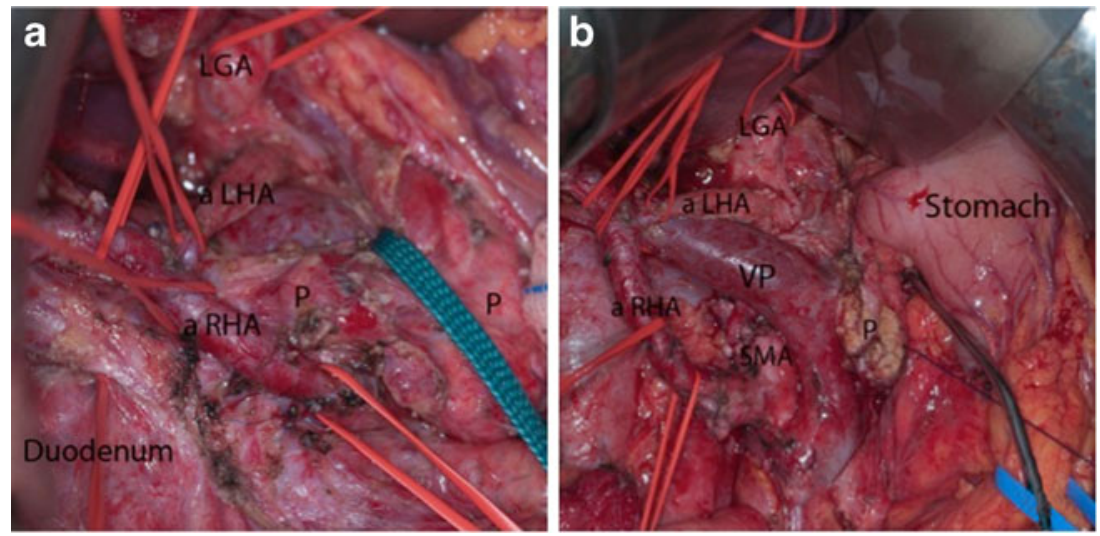
to predict resectability of pancreas head tumors in $80 \%$ of cases. $^{8}$

The other interesting feature of this case is the prepancreatic course of the aRHA, instead of the classically described retropancreatic and latero-cave course. ${ }^{5}$

In the presence of an aRHA, several options are possible for the surgeon, but there is a risk of arterial injury during the dissection if this is not recognized before or during the intervention. One problem is to dissect the artery along its whole course with a risk of vascular injury. Other strategies suggest a simple ligature of the artery if the clamping test shows a satisfactory hepatic vascularization with the use of ultrasonography. ${ }^{9}$ There is however a risk of long-term biliary ischemia, or even necrosis of a hepatic segment, or a risk of bile leak on the biliojejunostomy if the vascular supply is due to the aRHA. ${ }^{7}$ On the other hand, this risk is still debated. ${ }^{7}$ Others propose the reimplantation of the aRHA on the gastroduodenal artery to limit the ischemic risk, but this technique has been claimed to increase the risk of lethal hemorrhage in case of postoperative pancreatic fistula. ${ }^{9}$

\section{References}

1. Haller A. Icones anatomicae in quibus aliquae partes corporis humani delineatae proponuntur et arteriarum potissimum historia continetur. Vandenhoeck, Göttingen 1756

2. Michels NA. Newer anatomy of the liver and its variant blood supply and collateral circulation. Am J Surg 1966;112:337-347.

3. Hiatt JR, Gabbay J, Busuttil RW. Surgical anatomy of the hepatic arteries in 1000 cases. Ann Surg 1994;220:50-52.

4. Adamthwaite JA, Pennington N, Menon KV. Anomalous hepatic arterial anatomy discovered during pancreaticoduodenectomy. Surg Radiol Anat 2007;29:269-271.

5. Balachandran A, Darden DL, Tamm EP, Faria SC, Evans DB, Chamsangavej C. Arterial variants in pancreatic adenocarcinoma. Abdom Imaging 2008:33;214-221.

6. Dumitrascu T, David L, Popescu I. Posterior versus standard approach in pancreatoduodenectomy: a case-match study. Langenbecks Arch Surg 2010;395:677-684.

7. Chamberlain RS, El-Sedfy A, Rajkumar D. Aberrant hepatic arterial anatomy and Whipple procedure: Lessons learned. Am Surg 2011;77:517-526.

8. Spanknebel K, Conlon KC. Advances in the surgical management of pancreatic cancer. Cancer J 2001;7:312-323.

9. Yamamoto S, Kubota K, Rokkaku K, Nemoto T, Sakuma A. Disposal of replaced common hepatic artery coursing within the pancreas during pancreatoduodenectomy: Report of a case. Surg Today 2005;35:984-987. 